\title{
Discounted cash-flows analysis: An interactive fuzzy arithmetic approach
}

\author{
CÉDRIC LESAGE*
}

\begin{abstract}
Many models used in management and economics are based on an arithmetic basis. Moreover, the imperfection of generally available information in these disciplines has led to numerous decision support systems based on fuzzy arithmetic. However, their practical use did not have success expected, because their output were generally too imperfect to help a manager with relevant information. This paper outlines a computational method for decreasing the imperfection of the output of fuzzy arithmetic models. We suggest an approach aiming at integrating into the arithmetic models fuzzy information still not taken into account: the vague knowledge of the interaction potentially existing between the two variables of an arithmetic operation (addition, subtraction, division or product). Thanks to the modelling of three standard relations, we propose a solution towards an interactive fuzzy arithmetic, which reduces the imperfection of the output to help the decision maker. We apply this approach to the choice of investment by the criterion of the Net Present Value resulting from a Discounted Cash-Flows Analysis.
\end{abstract}

Classification Codes: M21, C63.

\section{Introduction}

Since the 70s-80s, many fuzzy logic-based models in management or economics have been developed in research literature. These theoretical works were grounded on the following motivation: fuzzy logic is a more relevant mathematical framework to deal with the kind of imperfect information generally available in management or economics: uncertain and imprecise (Gil Aluja, 1995). For instance, forecasting in management is more often confronted with judgements than with accurate statistical data.

However, a major obstacle seems to have hindered concrete application by the enterprises of these theoretical works: the output imperfection. Namely, fuzzy-input-based models generate automatically a fuzzy output, which may be useless in a decisionmaking process. Part of that imperfection comes from the inputs - the so-called "natural

\footnotetext{
* Centre de Recherche Rennais en Économie et en Gestion (UMR CNRS C6585), 11 rue Jean Macé, CS 70803, 35008 Rennes Cedex 7, France. E-mail: Cedric.Lesage@univ-rennes1.fr

Keywords: Fuzzy logic, fuzzy arithmetic, interactivity, discounted cash flows.
} 
imperfection". But another part is due to the use of the fuzzy operators, and is designed as the "artificial imperfection". Arithmetic models particularly, very much used in economics and management, generate an important artificial fuzziness harmful for the relevance of the output information.

The approach described in this paper aims at the decrease of artificial imperfection in fuzzy arithmetic models. We apply this approach on the Discounted Cash-Flow Analysis. We shall begin by presenting this widely management tool used into finance, accounting and decision problems (Sect. 2). Then, after a short presentation of basic fuzzy arithmetic (Sect. 3), we will propose a means of decreasing imperfection by modelling an available knowledge hardly taken into account: the interactivity between variables (Sect. 4). Finally we will present an illustrative application on the discounted cash-flows analysis (Sect. 5).

\section{The DCF model}

\subsection{Presentation}

The Discounted Cash-Flows (DCF) method appears to be the most popular methodology for assessing the economic value of investments. Investing means spending money today (the invested capital) in order to recover revenues after several years that the net balance will be positive. As more than one year is involved, the effect of time on this net value must be taken into account. The criterion of the Net Present Value, based on the Discounted Cash-Flow Analysis, is a very useful tool of decision-making. A common formulation stands as follows:

$$
N P V=\sum_{t=0}^{n} \frac{F t}{(1+r)^{t}}
$$

with

$N P V$ : Net Present Value,

$F t$ : Revenues - Cost and/or Investments,

$r$ : Discount rate, representing the expected profitability of the investment, as given the risk level assumed.

This approach, which is very efficient and consistent with the whole financial theory, raises some problems: the evaluation of the variables and the dependency between variables.

\subsection{The significance of relations for modeling}

There exist many kinds of relations between variables of economic and management models. Generally managers are inclined to neglect the relations of dependency between the variables. Many investment decision-making analyses suffer from such a drawback. However, those relations do exist and may have a significant effect on the choice. The following example illustrates that problem (taken from J.P. Frenois, 2001): sales are the 


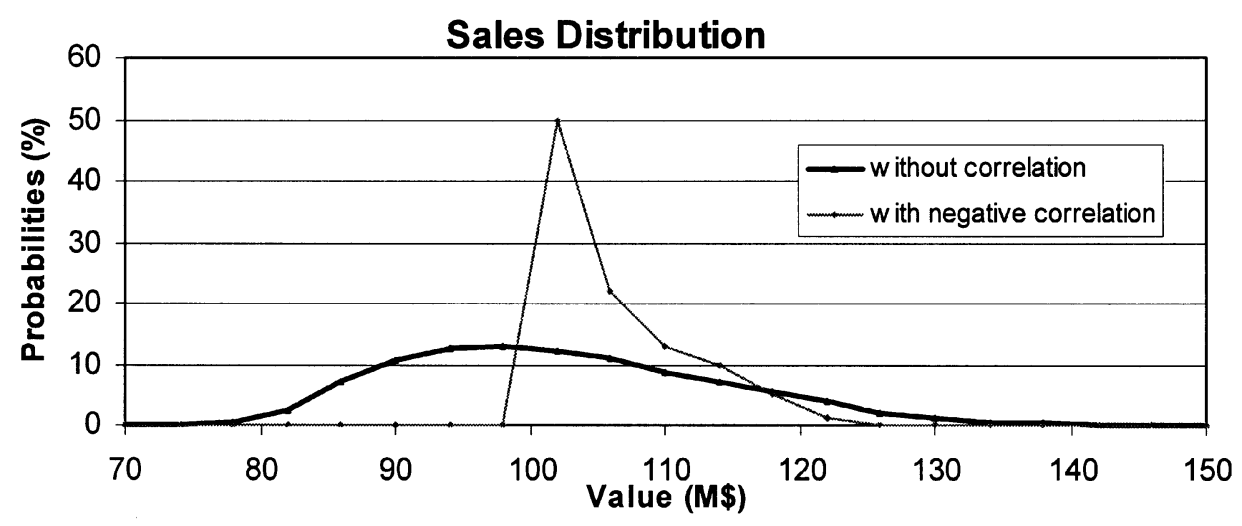

\begin{tabular}{|l|c|c|c|c|}
\hline & Minimum & Maximum & Average & Standard deviation \\
\hline Without correlation & 78 & 149 & 104 & 12 \\
\hline With negative correlation & 99 & 119 & 104 & 5 \\
\hline
\end{tabular}

* Source: adapted from Frénois, 2001, p. 19

Fig. 1. Comparison of probability of sales with and without correlation.

product of a quantity and a price. But quantity and price are not independent variables: the higher the price the lower the global demand and the sold quantity. If that relation is considered, the volatility of sales decreases, as illustrated by Figure 1. The first histogram represents the sales distribution of the new product, when no relation between price and quantity exists, while the second histogram illustrates that distribution when correlation is -1 . Even if the averages are equal, both distributions are very different: standard deviation has decreased by $-58 \%$. Ignoring negative correlations between two positive (or two negative) variables leads to an overestimation of the volatility of the project. Ignoring positive correlation has the opposite effect.

This problem is due to the frequent impossibility to statistically calculate the correlation coefficient. Therefore the calculation of the forecast cash flows and discount rate over a period of several years is difficult, even impossible, to be determined precisely. That is the reason why many models have been then developed under the fuzzy logic mathematical framework.

\section{Fuzzy Discounted Cash-Flows}

The fuzzy set theory was introduced by Zadeh (1965) to deal with problems in which a source of vagueness is involved. The basic concepts of fuzzy sets and arithmetic operations are briefly introduced (a complete presentation may be found in Klir, Yuan (1995)), followed by their application to the DCF models. 


\subsection{Basic Fuzzy Arithmetic concepts}

A fuzzy set can be defined mathematically by assigning to each possible individual in the universe of discourse a value representing its grade of membership in the fuzzy set. This grade corresponds to the degree to which individual is compatible with the concept represented by the fuzzy set. The membership grades are usually represented by real numbers in the $[0,1]$ interval (Fig. 2).

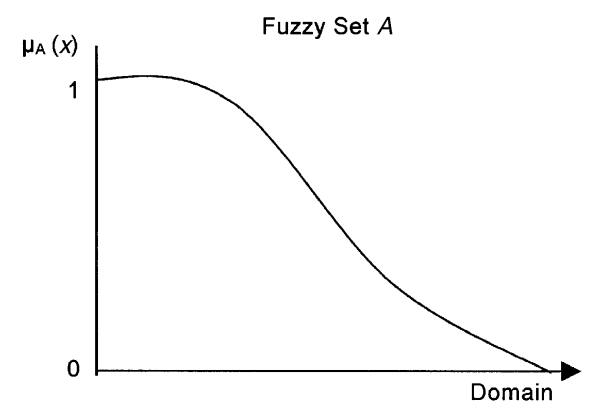

Fig. 2. A Fuzzy Set.

A special case of fuzzy sets is widely used: if a fuzzy set $A$, with the associated membership function $\mu_{A}(x)$ defined on $\Re$, which is normal $\left(\sup _{x} \mu_{A}(x)=1\right)$ and convex $\left(\forall x, y \in \Re\right.$ and $\forall \lambda \in[0,1], \mu_{A}(\lambda x+(1-\lambda) y) \geq \min \left(\mu_{A}(x), \mu_{A}(y)\right)$ then $A$ is a fuzzy number. A fuzzy number is a trapezoidal fuzzy number (TFN) if its membership function can be represented as (see Fig. 3 for an illustration):

$$
\mu_{A}(x)=\left\{\begin{array}{lc}
0, & x<L_{0} \\
\left(x-L_{0}\right) /\left(L_{1}-L_{0}\right), & L_{0} \leq x \leq L_{1} \\
1, & L_{1} \leq x \leq U_{1} \\
\left(U_{0}-x\right) /\left(U_{1}-U_{0}\right), & U_{1} \leq x \leq U_{0} \\
0, & x>U_{0}
\end{array}\right\} .
$$

Each TFN is obtained by asking the manager the values of the core $\left[L_{1}-U_{1}\right]$ (the most probable values) and those of the support $\left[L_{0}-U_{0}\right]$ (the lower and upper values), according to the method known as the experts' method (Aladenise, Bouchon-Meunier, 1997).

Then, the formulae of classic fuzzy arithmetic are applied to the TFN $\left(L_{0}, L_{1}, U_{1}, U_{0}\right)$. Zadeh's extension principle is now used to calculate membership function after mapping fuzzy sets through a function. The extension principle is defined as follows:

Let fuzzy sets $A_{1}, A_{2}, \ldots A_{n}$ be defined on the universes $X_{1}, X_{2}, \ldots, X_{n}$. The mapping for this input sets can be now defined as $B=\mu\left(A_{1}, A_{2}, \ldots A_{n}\right)$, where the membership function of the image $B$ is expressed as:

$$
\mu_{B}(y)=\max \left(\min \mu_{A_{1}}\left(x_{1}\right), \mu_{A_{2}}\left(x_{2}\right), \ldots \mu_{A_{n}}\left(x_{n}\right)\right) .
$$




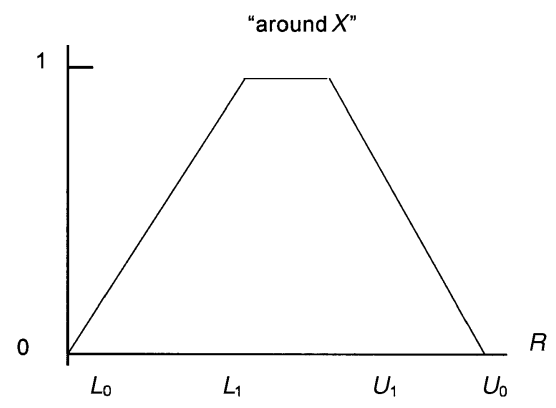

Fig. 3. A Trapezoidal Fuzzy Number (TFN).

This principle enables us to extend the classic arithmetical operations to fuzzy numbers:

Given: $X$, fuzzy number defined by $\left\{i, \mu_{x}(i)\right\}, Y$, fuzzy number defined by $\left\{j, \mu_{y}(j)\right\}$ then:

$$
\mu_{x * y}\left(i^{*} j\right)=\max _{i, j}\left(\min \mu_{x}(i), \mu_{y}(j)\right)
$$

where: $*$ is one of the following operations:,,$+- \times, /$, min, max.

When applied to $T F N$ s, formula (1) leads to easy arithmetic calculation:

Given $X, T F N$, defined by $\left(L_{0} x, L_{1} x, U_{1} x, U_{0} x\right) ; Y, T F N$, defined by $\left(L_{0} y, L_{1} y, U_{1} y, U_{0} y\right)$,

Then:

$X^{*} Y$ is a $T F N$, defined by $\left(L_{0}\left(x^{*} y\right), L_{1}\left(x^{*} y\right), U_{1}\left(x^{*} y\right), U_{0}\left(x^{*} y\right)\right)$,

such as:

$$
\begin{aligned}
& L_{0}\left(x^{*} y\right)=\min \left(L_{0} x^{*} L_{0} y, L_{0} x^{*} U_{0} y, U_{0} x^{*} L_{0} y, U_{0} x^{*} U_{0} y\right) \\
& L_{1}\left(x^{*} y\right)=\min \left(L_{1} x^{*} L_{1} y, L_{1} x^{*} U_{1} y, U_{1} x^{*} L_{1} y, U_{1} x^{*} U_{1} y\right) \\
& U_{1}\left(x^{*} y\right)=\max \left(L_{1} x^{*} L_{1} y, L_{1} x^{*} U_{1} y, U_{1} x^{*} L_{1} y, U_{1} x^{*} U_{1} y\right) \\
& U_{0}\left(x^{*} y\right)=\max \left(L_{0} x^{*} L_{0} y, L_{0} x^{*} U_{0} y, U_{0} x^{*} L_{0} y, U_{0} x^{*} U_{0} y\right)
\end{aligned}
$$

where: $*$ is one of the following operations:,,$+- \times, /$, min, max.

Figure 4 illustrates formula (2): the arithmetic operations on fuzzy numbers generalize interval analysis.

Many Fuzzy Discounted Cash-Flows models (based on first Buckley's paper, 1986) have been developed as an alternative to the conventional models, where either deterministic or probabilistic cash flows and discount rates are used.

$$
\tilde{N P V}=\sum_{t=0}^{n} \frac{\tilde{F} t}{(1+\tilde{r})^{t}}
$$




$\begin{array}{ccc}\text { "around 2" } & \text { "around 5" } & \text { "around 7" } \\ (0,5 ; 1,5 ; 2,5 ; 3,5) & (3,5 ; 5 ; 6,5) & (4 ; 6,5 ; 7,5 ; 10)\end{array}$

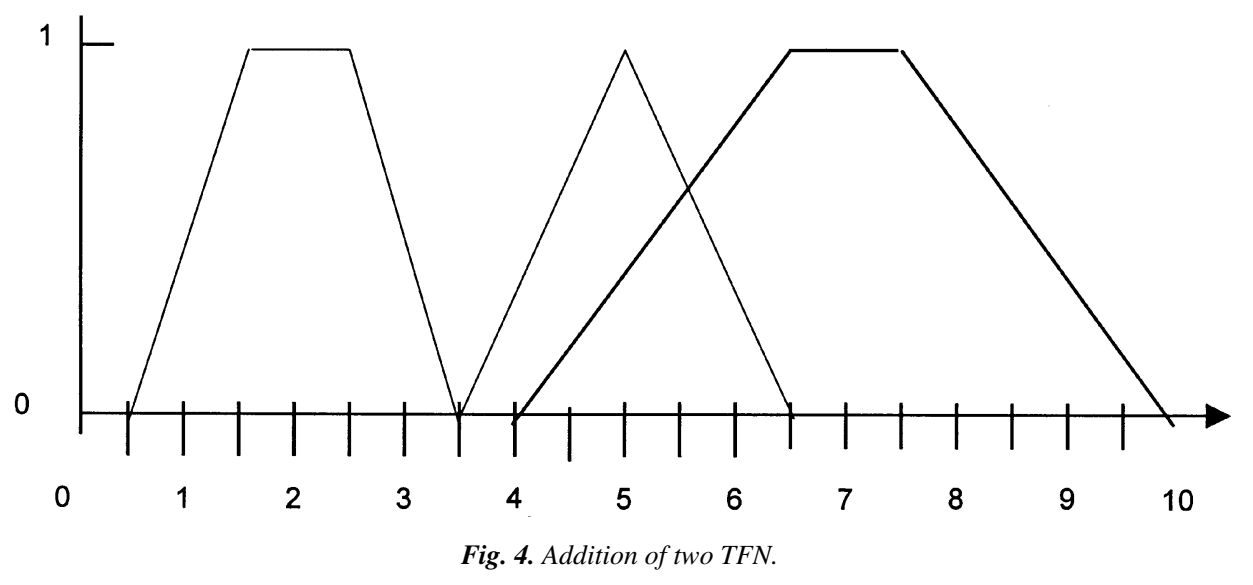

Fuzzy logic enables us to assess fuzzy data that take better into account of the imprecision and uncertainty of human judgements about forecast cash flows and discount rates. But this model (as well all derived models, e.g. Karsak, Tolga, 2001) is based on a sequence of fuzzy arithmetic operations, which generates artificial imperfection.

Namely a strong drawback of models based on basic fuzzy arithmetic must be stressed: these models do not consider error compensation and some technical approximations increases the artificial imperfection (Dubois, Prade, 1985, p. 23).

One point is frequently put aside: formula (1) is valid only if both variables are noninteractive (Dubois, Prade, 1985). In the opposite case, the general formulation is the following one:

$$
\mu_{x * y}\left(i^{*} j\right)=\max _{i, j}\left(\min \mu_{x}(i), \mu_{y}(j), \mu_{x, y}(i, j)\right) .
$$

The "non-interactivity" condition means that all couples $(x, y)$ are assessed, because no specific relation $\mathrm{R}$ between $X$ and $Y$ is assumed: then $\mathrm{R}(x, y)=1, \forall x, y \in \mathfrak{R}$. But some combinations may not have a real sense, because $x$ and $y$ may not occur together.

Now such an imperfection may be harmful for decision-making purposes. From a management perspective, the reduction of imperfection constitutes a top priority. For instance, the cash flows and the discount rate being regarded as non-interactive, the model must take all combinations into account, even if they have non-sense in reality. For a decision-making utilisation, this artificial fuzziness may be prejudicial, because it increases the number of alternative decisions. The significance of the relation between the variables in management leads us to suggest a way of dealing with interactivity into fuzzy arithmetic models as presented in the following section.

\section{Proposal for an Interactive Fuzzy Arithmetic}

This section presents an Interactive Fuzzy Arithmetic (IFA). This approach aims at reconciling both the computational advantage of the TFNs and the decrease of the output 
through the interactivity. After the presentation of the general process on specific relations, generalizations are explored.

\subsection{Basic functions}

Generally, managers do not know the precise relation existing between the variables. However, they know whether this relation belongs to one vague category such as a noninteractive, an increasing or a decreasing profile. This kind of information is very often the only available data.

Therefore, we will start by studying three standard relations:

Let's define: $X, Y T N F$, a relation $\mathrm{R}(X, Y)$, and the operator *, designing,,$+- \times, /$.

- $\mathrm{R}(X, Y)$ is non interactive: no specific relation between $X$ and $Y$ is assumed,

- $\mathrm{R}(X, Y)$ is increasing: $X$ is an increasing function of $Y$,

- $\mathrm{R}(X, Y)$ is decreasing: $X$ is a decreasing function of $Y$.

If the Non-INTERACTIVE relation is put aside (for which $\mu(x, y)=1$, for any $x \in X$ and any $y \in Y$ ), the problem is then to model the two other categories.

The starting point of our approach is illustrated by Figure 5. It is based on a simple positioning of the core and the support of the relation.

Increasing Relation $(X, Y)$

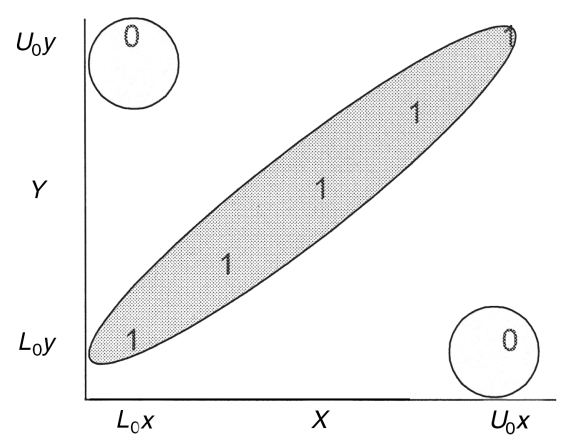

Decreasing Relation $(X, Y)$

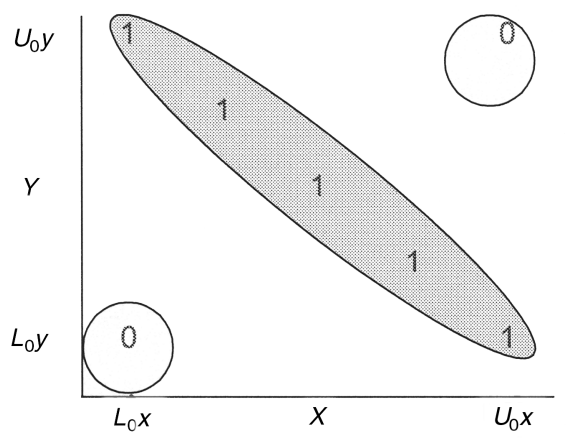

Fig. 5. Basis of the IFA.

For instance, in a case of an increasing function, couples $(x, y)$, with $x$ near $L_{0} x$ and $y$ near $U_{0} y$ have a null possibility. Conversely, couples $(x, y)$, with $x$ near $U_{0} x$ and $y$ near $U_{0} y$ are fully possible. The difficulty lies in the meaning of «near», which raised the question of the segmentation of the support of each variable. A necessary condition for the standard relations must be filled: no particular area of the matrix should be privileged, which means that the segmentation process must preserve symmetry.

\subsubsection{Basic structure}

Decreasing the imperfection of the output by using the relations between the inputs means that we intend to diminish the support and/or the core of the relation $\mathrm{R}(X, Y)$, in order to limit the number of possible combinations $(x, y)$. We decided to segment the 
support of each variable to have a set of values that will be then combined. We adopted a segmentation into 10 categories, which may facilitate calculations and accelerate the processing time (Convention 1).

\begin{tabular}{|c|}
\hline Convention 1 \\
\hline Segmentation of each support into 10 categories \\
\hline
\end{tabular}

Naturally, those 10 categories define, for each variable, 11 bound-values $B x_{i}$, $i=\{1,2, \ldots 11\}$. For each kind of function (increasing or decreasing), the positioning of 0 -values and of the 1-diagonal is given by Convention 2:

\begin{tabular}{|c|c|}
\hline & Convention 2 \\
\hline \multicolumn{2}{|c|}{$\mathrm{R}(X, Y)$ is an ascending function } \\
\hline Support : 0 -values & Core: 1-values \\
\hline $\begin{array}{l}\mu_{\mathrm{R}(X, Y)}\left(B x_{1} ; B y_{11}\right)=0 \\
\mu_{\mathrm{R}(X, Y)}\left(B x_{11} ; B y_{1}\right)=0\end{array}$ & $\begin{array}{l}\mu_{\mathrm{R}(X, Y)}\left(B x_{i} ; B y_{i}\right)=1 \text { for } i=\{1,2, \ldots, 11\} \\
\mu_{\mathrm{R}(X, Y)}\left(B x_{i} ; B y_{i+1}\right)=1 \text { for } i=\{1,2, \ldots, 10\} \\
\mu_{\mathrm{R}(X, Y)}\left(B x_{i+1} ; B y_{i}\right)=1 \text { for } i=\{1,2, \ldots, 10\}\end{array}$ \\
\hline \multicolumn{2}{|c|}{$\mathrm{R}(X, Y)$ is an descending function } \\
\hline Support: 0 -values & Core: 1-values \\
\hline $\begin{array}{c}\mu_{\mathrm{R}(X, Y)}\left(B x_{1} ; B y_{1}\right)=0 \\
\mu_{\mathrm{R}(X, Y)}\left(B x_{11} ; B y_{11}\right)=0\end{array}$ & $\begin{array}{l}\mu_{\mathrm{R}(X, Y)}\left(B x_{i} ; B y_{12-i}\right)=1 \text { for } i=\{1,2, \ldots, 11\} \\
\mu_{\mathrm{R}(X, Y)}\left(B x_{i} ; B y_{11-i}\right)=1 \text { for } i=\{1,2, \ldots, 10\} \\
\mu_{\mathrm{R}(X, Y)}\left(B x_{i+1} ; B y_{12-i}\right)=1 \text { for } i=\{1,2, \ldots, 10\}\end{array}$ \\
\hline
\end{tabular}

Figure 6 illustrates Convention 2 for both relations.

\subsubsection{Matrix of combining values}

On a second step, values $L_{1}$ and $U_{1}$ defining the core of each variable, are added to the 11 bound-values of the basic structure. These values are included on each axis, with a rank corresponding to their value. They do not modify the basic structure, but enable the model to increase the number of combinations $X^{*} Y$. This method gives the advantage of increasing the robustness of the output and enables us to consider the BFA approach as a specific case of the IFA, with $\mathrm{R}(X, Y)$ non interactive.

The membership of these values to $\mathrm{R}(\mathrm{X}, \mathrm{Y})$ is obtained by the extension of the basic structure, according to Convention 3 :

Convention 3

If $\exists i$ such as: $L_{1} x \in\left[B x_{i} ; B x_{i+1}\left[\right.\right.$, then Convention 2 is extended to $L_{1} x$ on a $B x_{i}$ basis

If $\exists i$ such as: $\left.U_{1} x \in\right] B x_{i} ; B x_{i+1}$, then Convention 2 is extended to $U_{1} x$ on a $B x_{i+1}$ basis 


\begin{tabular}{|c|c|c|c|c|c|c|c|c|c|c|c|}
\hline \multicolumn{12}{|c|}{ Increasing Relation $(X, Y)$} \\
\hline$B y_{11}$ & 0 & & & & & & & & & 1 & 1 \\
\hline$B y_{10}$ & & & & & & & & & 1 & 1 & 1 \\
\hline$B y_{9}$ & & & & & & & & 1 & 1 & 1 & \\
\hline$B y_{8}$ & & & & & & & 1 & 1 & 1 & & \\
\hline$B y_{7}$ & & & & & & 1 & 1 & 1 & & & \\
\hline$B y_{6}$ & & & & & 1 & 1 & 1 & & & & \\
\hline$B y_{5}$ & & & & 1 & 1 & 1 & & & & & \\
\hline$B y_{4}$ & & & 1 & 1 & 1 & & & & & & \\
\hline$B y_{3}$ & & 1 & 1 & 1 & & & & & & & \\
\hline$B y_{2}$ & 1 & 1 & 1 & & & & & & & & \\
\hline \multirow[t]{2}{*}{$B y_{1}$} & 1 & 1 & & & & & & & & & 0 \\
\hline & $B x_{1}$ & $B x_{2}$ & $B x_{3}$ & $B x_{4}$ & $B x_{5}$ & $B x_{6}$ & $B x_{7}$ & $B x_{8}$ & $B x_{9}$ & $B x_{10}$ & $B x_{11}$ \\
\hline
\end{tabular}

\begin{tabular}{|c|c|c|c|c|c|c|c|c|c|c|c|}
\hline \multicolumn{12}{|c|}{ Decreasing Relation } \\
\hline$B y_{11}$ & 1 & 1 & & & & & & & & & 0 \\
\hline$B y_{10}$ & 1 & 1 & 1 & & & & & & & & \\
\hline$B y_{9}$ & & 1 & 1 & 1 & & & & & & & \\
\hline$B y_{8}$ & & & 1 & 1 & 1 & & & & & & \\
\hline$B y_{7}$ & & & & 1 & 1 & 1 & & & & & \\
\hline$B y_{6}$ & & & & & 1 & 1 & 1 & & & & \\
\hline$B y_{5}$ & & & & & & 1 & 1 & 1 & & & \\
\hline$B y_{4}$ & & & & & & & 1 & 1 & 1 & & \\
\hline$B y_{3}$ & & & & & & & & 1 & 1 & 1 & \\
\hline$B y_{2}$ & & & & & & & & & 1 & 1 & 1 \\
\hline \multirow[t]{2}{*}{$B y_{1}$} & 0 & & & & & & & & & 1 & 1 \\
\hline & $B x_{1}$ & $B x_{2}$ & $B x_{3}$ & $B x_{4}$ & $B x_{5}$ & $B x_{6}$ & $B x_{7}$ & $B x_{8}$ & $B x_{9}$ & $B x_{10}$ & $B x_{11}$ \\
\hline
\end{tabular}

Fig. 6. Basic structure of the relations. 
The application of the three conventions leads to a $13 \times 13$ matrix and whose elements are the 0 -valued and 1-valued membership degrees of the relation $\mathrm{R}(X, Y)$. (See Fig. 7 for an example).

Example: Let's suppose two variables: Price: $X=(3.00 ; 3.05 ; 3.10 ; 3.20)$ and Quantity: $Y=(230 ; 250 ; 260$; 270), and a decreasing relation between $X$ and $Y$.

\begin{tabular}{|c|c|c|c|c|c|c|c|c|c|c|c|c|c|c|}
\hline 270 & $B y_{11}$ & 1 & 1 & & & & & & & & & & & 0 \\
\hline 266 & $B y_{10}$ & 1 & 1 & 1 & 1 & & & & & & & & & \\
\hline 262 & $B y_{9}$ & & 1 & 1 & 1 & 1 & & & & & & & & \\
\hline 260 & $U 1 y$ & & 1 & 1 & 1 & 1 & & & & & & & & \\
\hline 258 & $B y_{8}$ & & & 1 & 1 & 1 & 1 & & & & & & & \\
\hline 254 & $B y_{7}$ & & & & & 1 & 1 & 1 & 1 & & & & & \\
\hline 250 & $L 1 y$ & & & & & & 1 & 1 & 1 & 1 & & & & \\
\hline 250 & $B y_{6}$ & & & & & & 1 & 1 & 1 & 1 & & & & \\
\hline 246 & $B y_{5}$ & & & & & & & 1 & 1 & 1 & 1 & & & \\
\hline 242 & $B y_{4}$ & & & & & & & & & 1 & 1 & 1 & & \\
\hline 238 & $B y_{3}$ & & & & & & & & & & 1 & 1 & 1 & \\
\hline 234 & $B y_{2}$ & & & & & & & & & & & 1 & 1 & 1 \\
\hline \multirow[t]{3}{*}{230} & $B y_{1}$ & 0 & & & & & & & & & & & 1 & 1 \\
\hline & & $B x_{1}$ & $B x_{2}$ & $B x_{3}$ & $L 1 x$ & $B x_{4}$ & $B x_{5}$ & $U 1 x$ & $B x_{6}$ & $B x_{7}$ & $B x_{8}$ & $B x_{9}$ & $B x_{10}$ & $B x_{11}$ \\
\hline & & 3.00 & 3.02 & 3.04 & 3.05 & 3.06 & 3.08 & 3.10 & 3.10 & 3.12 & 3.14 & 3.16 & 3.18 & 3.20 \\
\hline
\end{tabular}

Convention 1 gives the $X$ - and $Y$-axes.

Convention 2 gives the general structure of the decreasing relation.

Convention 3 gives the following extensions (marked in grey):

$L_{1} X$ : considered as $B x_{3}$ and $U_{1} X$ : considered as $B x_{6}$;

$L_{1} Y$ : considered as $B y_{6}$ and $U_{1} Y$ : considered as $B y_{9}$.

Fig. 7. Set of combining values.

\subsubsection{Support and core of the relation $R(X, Y)$}

The matrix of the combining values enables us to define the support and the core of the relation $\mathrm{R}(X, Y)$.

Let's define $\mathcal{N}=\{1,2, \ldots 13\}$. Then:

- The support of $X^{*} Y$, noted $\left[L_{0}\left(X^{*} Y\right), U_{0}\left(X^{*} Y\right)\right]$, is given by the following formula: Let's define: $S=\left\{B x_{i}^{*} B y_{j} / \mu_{\mathrm{R}(X, Y)}\left(B x_{i} ; B y_{j}\right)>0\right\} \quad \forall i \in \mathcal{N}, \forall j \in \mathcal{N}$. 
Then:

$$
L_{0}\left(x^{*} y\right)=\min _{i, j}(S) \text { and } U_{0}\left(x^{*} y\right)=\max _{i, j}(S)
$$

- The core of $X^{*} Y$, noted $\left[L_{1}\left(X^{*} Y\right), U_{1}\left(X^{*} Y\right)\right]$, is given by the following formula:

Let's define: $C=\left\{B x_{i}^{*} B y_{j} / L_{1} x \leq B x_{i} \leq U_{1} x ; L_{1} y \leq B y_{j} \leq U_{1} y ; \mu_{\mathrm{R}(X, Y)}\left(B x_{i} ; B y_{j}\right)=1\right\}$ $\forall i \in \mathcal{N}, \forall j \in \mathcal{N}$.

Then:

$$
L_{1}\left(x^{*} y\right)=\min _{i, j}(C) \text { and } U_{1}\left(x^{*} y\right)=\max _{i, j}(C)
$$

\begin{tabular}{|c|c|c|c|c|c|c|c|c|c|c|c|c|c|c|}
\hline 270 & $B y_{11}$ & 810 & 815.4 & 820.8 & 823.5 & 826.2 & & & 837 & 842.4 & 847.8 & 853.2 & 858.6 & \\
\hline 266 & $B y_{10}$ & 798 & 803.3 & 808.6 & & & & & 824.6 & 829.9 & 835.2 & 840.6 & 845.9 & 851.2 \\
\hline 262 & $B y_{9}$ & 786 & 791.2 & 796.5 & & & & & 812.2 & 817.4 & 822.7 & 827.9 & 833.2 & 838.4 \\
\hline 260 & $2 x$ & 780 & 7852 & 7904 & & & & & 806 & $8 \times 2$ & 8164 & $82 \times 6$ & 826.8 & 832 \\
\hline 258 & Bes & $7 x$ & $7 \times 2$ & 7843 & & & & & 7988 & 805 & $8 \mathrm{~K} 1$ & 815,3 & 820.4 & 825.6 \\
\hline 254 & $B_{4}$ & 782 & $76 \times 1$ & 7722 & & & & & $78 x 4$ & 7925 & $79 \times 6$ & 8026 & $80 \times 7$ & 8128 \\
\hline 250 & $\Delta x$ & 750 & 755 & & & & & & $7 \times 5$ & 78 & 78 & 798 & 78 & $80 Q$ \\
\hline 250 & $B y_{6}$ & 750 & 755 & 760 & & & & & 775 & 780 & 785 & 790 & 795 & 800 \\
\hline 246 & $B y_{5}$ & 738 & 742.9 & 747.8 & & & & & 762.6 & 767.5 & 772.4 & 777.4 & 782.3 & 787.2 \\
\hline 242 & $B y_{4}$ & 726 & 730.8 & 735.7 & & & & & 750.2 & 755 & 759.9 & 764.7 & 769.6 & 774.4 \\
\hline 238 & $B y_{3}$ & 714 & 718.8 & 723.5 & & & & & 737.8 & 742.6 & 747.3 & 752.1 & 756.8 & 761.6 \\
\hline 234 & $B y_{2}$ & 702 & 706.7 & 711.4 & & & & & 725.4 & 730.1 & 734.8 & 739.4 & 744.1 & 748.8 \\
\hline \multirow[t]{3}{*}{230} & $B y_{1}$ & & 694.6 & 699.2 & & & & & 713 & 717.6 & 722.2 & 726.8 & 731.4 & 736 \\
\hline & & $B x_{1}$ & $B x_{2}$ & $B x_{3}$ & & & & & $B x_{6}$ & $B x_{7}$ & $B x_{8}$ & $B x_{9}$ & $B x_{10}$ & $B x_{11}$ \\
\hline & & 3.00 & 3.02 & 3.04 & & & 3 & & 3.10 & 3.12 & 3.14 & 3.16 & 3.18 & 3.20 \\
\hline
\end{tabular}

An illustrative example is given in Figure 8.

\begin{tabular}{|l|l|l|l|l|}
\hline & $x \times y / \mu_{\mathrm{R}(X, Y)}(x, y)=1$ & $x / \mu_{x}(x)=1$ & $y / \mu_{y}(y)=1$ \\
\hline
\end{tabular}

The values $x \times y$ with a 0 -membership degree have been suppressed.

$$
L_{0}\left(x^{*} y\right)=\min _{i, j}(S)=694.6 \quad U_{0}\left(x^{*} y\right)=\max _{i, j}(S)=858.6
$$

$L_{1}\left(x^{*} y\right)=\min _{i, j}(C)=770 \quad U_{1}\left(x^{*} y\right)=\max _{i, j}(C)=795.6$

Fig. 8. Illustrative example.

This approach is the basic IFA. The following sections give an evaluation and a generalization.

\subsection{Advantages and limits}

First, the main advantage of the calculation on the TFN given by formula (2) is that only four data $L_{0}, L_{1}, U_{1}, U_{0}$ are necessary. The IFA approach conserves this property. 
Then, we presented the IFA as an approach that reduces the artificial imperfection. What are the results?

In order to measure the decrease in imperfection, we suggest to use the following entropy (Dubois, Prade, 1988, p. 23): $\mathrm{E}=\Sigma_{i} \mu\left(x_{i}\right)$. Applied to a $T F N$, it corresponds to the area of the trapezoid: "Area Entropy" $A E=0.5\left(\left(U_{0}-L_{0}\right)+\left(U_{1}-L_{1}\right)\right)$.

With a few exceptions (for instance a decrease by $20 \%$ for the addition - resp. subtraction - of two triangular fuzzy numbers - i.e. a $T F N$ for which: $L_{1}=\mathrm{U}_{1}-$, with a decreasing - resp. increasing - relation) the decrease of imperfection on one operation cannot be a priori determined. Namely it depends on the following factors:

- the kind of arithmetic operation,

- the kind of function (increasing or decreasing),

- the positioning of the cores of variables $X$ and $Y$ on their axis of categories.

In practice, however, a decrease of imperfection between 30 and $50 \%$ is recorded when the IFA is applied to management models, that are usually made up numerous operations (See the application in section 5).

However, that approach raises a difficulty due to the normality of the output $X^{*} Y$. A fuzzy set $X$ is normal if there exists at least one element $x \in X$ such as $\mu(x)=1$. In real management contexts, this property makes certain that at least one solution is fully possible. It enables also the calculation process to have always TFNs as outputs that may be used as inputs for further operations. By nature, the BFA approach leads to normal solutions $X^{*} Y$. But when the IFA is used, this property may not be conserved: it is possible that no couple $\left(B x_{i}, B y_{j}\right)$ meets simultaneously the three conditions given by formula (5). This limit of the basic IFA approach will be suppressed by the generalization as presented in the following section.

\subsection{Generalization of the IFA}

It is possible to generalize the basic IFA approach to benefit from various profiles of relations. Namely, the number of incompatible combinations may be more or less significant depending on the nature of the relation between both variables of the operation. Then two management problems can differ only because the level of knowledge of the relations is different. For instance, for some periods in one year, there may exist a strong decreasing relation between price and quantity, whereas, for other periods, this relation is weaker, because the customers at that time are captive customers. The basic IFA does not take into account that diversity of knowledge levels.

The generalized IFA is based on various positioning on the matrix of the relation $\mathrm{R}(X, Y)$ of (as illustrated by Fig. 9$)$ :

- 0 -values: by bringing the diagonals of 0 -values nearer to the diagonal of 1 -values of the basic position, the number of possible combinations is decreased (the support of the relation), which leads to a decrease of imperfection of the output $X^{*} Y$;

- 1-values: by bringing the diagonals of 1 -values nearer to the position of the 0 -value of the basic position, the number of possible combinations is increased (the core of the relation), which leads to an increase of imperfection of the output $X^{*} Y$.

We then obtain, for each of both functions (increasing or decreasing), a continuum of 19 levels of knowledge, from total ignorance (level 0: non interactivity, which equals to 
Increasing imperfection by expanding the 1-values: Level $k=9$ to $k=0$ (non interactivity)

\begin{tabular}{|c|c|c|c|c|c|c|c|c|c|c|c|c|}
\hline$k=0$ & $B y_{11}$ & 0 & & & & & & & & & 1 & 1 \\
\hline$k=1$ & $B y_{10}$ & & & & & & & & & 1 & 1 & 1 \\
\hline$k=2$ & $B y_{9}$ & & & & & & & & 1 & 1 & 1 & \\
\hline$k=3$ & $B y_{8}$ & & & & VII & & & 1 & 1 & 1 & & \\
\hline$k=4$ & $B y_{7}$ & & & & & & 1 & 1 & 1 & & & \\
\hline$k=5$ & $B y_{6}$ & & & & & 1 & 1 & 1 & & & & \\
\hline$k=6$ & $B y_{5}$ & & & & 1 & 1 & 1 & & & & & \\
\hline$k=7$ & $B y_{4}$ & & & 1 & 1 & 1 & & & & & & \\
\hline$k=8$ & $B y_{3}$ & & 1 & 1 & 1 & & & & & & & \\
\hline \multirow[t]{4}{*}{$K \geq 9$} & $B y_{2}$ & 1 & 1 & 1 & & & & & & & & \\
\hline & $B y_{1}$ & 1 & 1 & & & & & & & & & 0 \\
\hline & & $B x_{1}$ & $B x_{2}$ & $B x_{3}$ & $B x_{4}$ & $B x_{5}$ & $B x_{6}$ & $B x_{7}$ & $B x_{8}$ & $B x_{9}$ & $B x_{10}$ & $B x_{11}$ \\
\hline & & & $k \geq 9$ & $k=8$ & $k=7$ & $k=6$ & $k=5$ & $k=4$ & $k=3$ & $k=2$ & $k=1$ & $k=0$ \\
\hline
\end{tabular}

Decreasing imperfection by expanding the 0 -values : Level $k=10$ to $k=18$

\begin{tabular}{|c|c|c|c|c|c|c|c|c|c|c|c|c|}
\hline$K \leq 9$ & $B y_{11}$ & 0 & & & & & & & & & 1 & 1 \\
\hline$k=10$ & $B y_{10}$ & & & & & & & & & 1 & 1 & 1 \\
\hline$k=11$ & $B y_{9}$ & 010 & & & & & & & 1 & 1 & 1 & \\
\hline$k=12$ & $B y_{8}$ & & & & & & & 1 & 1 & 1 & & \\
\hline$k=13$ & $B y_{7}$ & & & $L$ & & & 1 & 1 & 1 & & & \\
\hline$k=14$ & $B y_{6}$ & & & & & 1 & 1 & 1 & & & & \\
\hline$k=15$ & $B y_{5}$ & & & & 1 & 1 & 1 & & & & & \\
\hline$k=16$ & $B y_{4}$ & & & 1 & 1 & 1 & & & & & & \\
\hline$k=17$ & $B y_{3}$ & & 1 & 1 & 1 & & & & & & & \\
\hline \multirow[t]{4}{*}{$k=18$} & $B y_{2}$ & 1 & 1 & 1 & & & & & & & & \\
\hline & $B y_{1}$ & 1 & 1 & & & & & & & & & 0 \\
\hline & & $B x_{1}$ & $B x_{2}$ & $B x_{3}$ & $B x_{4}$ & $B x_{5}$ & $B x_{6}$ & $B x_{7}$ & $B x_{8}$ & $B x_{9}$ & $B x_{10}$ & $B x_{11}$ \\
\hline & & & $k=18$ & $k=17$ & $k=16$ & $k=15$ & $k=14$ & $k=13$ & $k=12$ & $k=11$ & $k=10$ & $K \leq 9$ \\
\hline
\end{tabular}

Fig. 9. The variations of an increasing function. 
the BFA approach) to the very precise knowledge (level 18: crisp linear relation), with level 9 corresponding to the basic IFA. We note $X *{ }^{(+k)} Y$ (resp. $X^{*(-k)} Y$ ) the operation * by the IFA of two TFN $X$ and $Y$ linked by an increasing relation (resp. decreasing) of level $k$.

Moreover, the generalized IFA enables us to settle the problem of normality: if one output $X^{*} Y$ has no core, for a given k-level of knowledge, then you try the $(k-1)$ level, until a core is found.

The precision proposed potentially by the available 19 levels of knowledge must not delude managers. One subject cannot discriminate a vague relation, for instance, of level 13 from another of level 14. The model must propose a scale with a small number of well-differentiated levels (see the example used for the DCF model in section 5). Moreover, this scale must be context-dependent, that is to say specifically adapted to the studied problem. Namely, in some cases, the average level of precision should be regarded as very demanding in other cases. The number and the smoothness of the 19 kinds of relations may allow the modeling of relations adapted to various contexts.

\section{Application}

Let's demonstrate the IFA approach on the FDCF via a numerical example. Let's suppose an investment decision making based on the criterion of the Net Present Value between two projects $X$ and $Y$.

\subsection{DCF Modeling}

Project $X$ is a middle-market product, with a price slightly high, a cost structure weighting variable costs. Project $Y$ is characterized by a mass-market product, sensitive to the price and produced in large quantities, demanding a higher initial investment, with a cost structure mainly based on fixed costs. The initial data are collected by the experts' method and are shown in Figures 10 and 11. Both projects are also different according to the relations between their variables. We used the following scale for the assessing of levels of knowledge of the relations:

\begin{tabular}{|l|l|l|l|l|l|}
\hline Linguistic scale & Very weak & Weak & Moderate & Strong & Very strong \\
\hline IFA -level & 0 & 3 & 6 & 9 & 12 \\
\hline
\end{tabular}

By using this table of correspondence, information collected amongst the managers leads to the following evaluation:

- Relation price-quantity

As product $Y$ is very sensitive to the price, the manager provides a very strong decreasing relation between both variables $(-12)$. As for product $X$, this decreasing relation is assessed at moderate $(-6)$.

- Relation revenues-charges

Charges include fixed and variable costs. The increasing relation between charges and revenues is then stronger for a structure with a strong proportion of variable costs. The manager assesses this relation at a strong level for $X(+9)$ and moderate $(+6)$ for $Y$. 
- Relation discount-rate-cash-flows

The discount rate used by a project takes a risk factor into account. Project $Y$ is a priori more risky than project $X$ (higher initial investment, higher forecasted level of sales, etc.). The manager assesses a weak increasing for project $X(+6)$ and a very strong increasing relation for project $Y(+12)$.

- Relation $C F_{n}$ and $C F_{n+1}$

Scenarios may differ from year to another: if one year is good, next year may be bad (saturation of demand), or, on the contrary, the next year may be best (phenomena of inertia). A relation between following years may exist. Let us suppose that the manager assesses that relation at weak increasing for $X(+3)$ and strong increasing for $Y(+9)$.

Then, the DCF model of each project stands as follows:

Project $X$ :

$\forall i \in\{1,2, \ldots, 5\}, D C F_{i}=\left(\left(Q_{i} \times{ }^{-6} P i\right)-{ }^{+9} C_{i}\right) /^{+6}\left(1+r_{i}\right)$

NPVX $=I_{0}+{ }^{+3} \mathrm{DCF}_{1}+{ }^{+3} \mathrm{DCF}_{2}+{ }^{+3} \mathrm{DCF}_{3}+{ }^{+3} \mathrm{DCF}_{4}+{ }^{+3} \mathrm{DCF}_{5}$

Project $Y$ :

$\forall i \in\{1,2, \ldots, 5\}, D C F_{i}=\left(\left(Q_{i} *-12 P_{i}\right)-{ }^{+6} C_{i}\right) /{ }^{+12}\left(1+r_{i}\right)$

NPV Y $=I_{0}+{ }^{+9} \mathrm{DCF}_{1}+{ }^{+9} \mathrm{DCF}_{2}+{ }^{+9} \mathrm{DCF}_{3}+{ }^{+9} \mathrm{DCF}_{4}+{ }^{+9} \mathrm{DCF}_{5}$.

\subsection{Evaluation}

The Net Present Value (NPV) calculated for each project by the classical formula makes both projects equivalent (Fig. 10: 96484 Euros for $X$ and 98082 Euros for $Y$ ). Nevertheless, this average value is not sufficient for a decision-making. This value may be reached according to various levels of risk that are specific to each project. The method of scenarios enables the manager to differentiate between the projects according to their level of risk. It generally assesses 3 kinds of scenarios:

- Probable scenario: it presents average values, as illustrated by the classical calculation.

- Pessimistic scenario: the $N P V$ is calculated by combining the most unfavorable values for each variable. These values correspond to the values $L_{0}$ of the TFN obtained

\begin{tabular}{|c|c|c|c|c|c|c|}
\hline & \multicolumn{3}{|c|}{ Investment $X$} & \multicolumn{3}{|c|}{ Investment $Y$} \\
\hline Initial Investment & 3500000 & CF0 & -4050000 & 4625000 & CF0 & -5375000 \\
\hline Average charge rate & $55 \%$ & $\mathrm{CF} 1$ & 875566 & $40 \%$ & CF1 & 1084843 \\
\hline Average sales increase rate & $7.5 \%$ & CF2 & 851795 & $12 \%$ & CF2 & 1089708 \\
\hline Quantity & 20000 & CF3 & 828669 & 20000 & CF3 & 1094594 \\
\hline Price & 100 & CF4 & 806171 & 90 & CF4 & 1099503 \\
\hline Discount rate & $10.5 \%$ & CF5 & 784284 & $11.5 \%$ & CF5 & 1104433 \\
\hline Classical NPV & & & 96484 & & & 98082 \\
\hline
\end{tabular}

Fig. 10. Initial Data. 
by the BFA approach. For instance, project $X\left(L_{0}=-3133934\right)$ is less risky than project $Y\left(L_{0}=-4693731\right)$ because the extreme loss is lower for $X$ than for $Y$.

- Optimistic scenario: the $N P V$ is calculated by combining the most favorable values for each variable. These values correspond to the values $\mathrm{U}_{0}$ of the $T F N$ obtained by the BFA approach (Fig. 12). For instance, project $X\left(U_{0}=3458590\right)$ gives the opportunity of a lower gain than project $Y\left(U_{0}=5411054\right)$.

Then, classical analysis tends to choose $X$ if you are risk advert, and $Y$ if you are risk seeker.

\begin{tabular}{|l|c|c|c|c|c|c|c|c|}
\hline Data for Fuzzy NPV & \multicolumn{3}{|c|}{ Investment $X$} & \multicolumn{3}{|c|}{ Investment $Y$} \\
\hline Scenario & $V . P$. & $P$. & $O$. & $V . O$. & $V . P$. & $P$. & $O$. & $V . O$. \\
\hline NFT values & $L_{0}$ & $L_{1}$ & $U_{1}$ & $U_{0}$ & $L_{0}$ & $L_{1}$ & $U_{1}$ & $U_{0}$ \\
\hline Rate of charges & & & & & & & & \\
\hline year 1 & $50.0 \%$ & $54.0 \%$ & $56.0 \%$ & $60.0 \%$ & $35.0 \%$ & $39.5 \%$ & $40.5 \%$ & $45.0 \%$ \\
\hline year 2 & $50.0 \%$ & $53.0 \%$ & $57.0 \%$ & $60.0 \%$ & $35.0 \%$ & $39.0 \%$ & $41.0 \%$ & $45.0 \%$ \\
\hline year 3 & $50.0 \%$ & $52.0 \%$ & $58.0 \%$ & $60.0 \%$ & $35.0 \%$ & $38.5 \%$ & $41.5 \%$ & $45.0 \%$ \\
\hline year 4 & $50.0 \%$ & $51.0 \%$ & $59.0 \%$ & $60.0 \%$ & $35.0 \%$ & $38.0 \%$ & $42.0 \%$ & $45.0 \%$ \\
\hline year 5 & $50.0 \%$ & $50.0 \%$ & $60.0 \%$ & $60.0 \%$ & $35.0 \%$ & $37.5 \%$ & $42.5 \%$ & $45.0 \%$ \\
\hline Annual evolution of sales & & & & & & & & \\
\hline year 1 & $5.0 \%$ & $7.0 \%$ & $8.0 \%$ & $10.0 \%$ & $5.0 \%$ & $11.0 \%$ & $13.0 \%$ & $19.0 \%$ \\
\hline year 2 & $5.0 \%$ & $6.5 \%$ & $8.5 \%$ & $10.0 \%$ & $5.0 \%$ & $10.5 \%$ & $13.5 \%$ & $19.0 \%$ \\
\hline year 3 & $5.0 \%$ & $6.0 \%$ & $9.0 \%$ & $10.0 \%$ & $5.0 \%$ & $10.0 \%$ & $14.0 \%$ & $19.0 \%$ \\
\hline year 4 & $5.0 \%$ & $5.5 \%$ & $9.5 \%$ & $10.0 \%$ & $5.0 \%$ & $9.5 \%$ & $14.5 \%$ & $19.0 \%$ \\
\hline year 5 & $5.0 \%$ & $5.0 \%$ & $10.0 \%$ & $10.0 \%$ & $5.0 \%$ & $9.0 \%$ & $15.0 \%$ & $19.0 \%$ \\
\hline Annual evolution of price & & & & & & & & \\
\hline year 1 & $-4.0 \%$ & $-1.0 \%$ & $1.0 \%$ & $4.0 \%$ & $-5.0 \%$ & $0.0 \%$ & $0.0 \%$ & $5.0 \%$ \\
\hline year 2 & $-4.0 \%$ & $-2.0 \%$ & $2.0 \%$ & $4.0 \%$ & $-5.0 \%$ & $-1.0 \%$ & $1.0 \%$ & $5.0 \%$ \\
\hline year 3 & $-4.0 \%$ & $-4.0 \%$ & $4.0 \%$ & $4.0 \%$ & $-5.0 \%$ & $-2.0 \%$ & $2.0 \%$ & $5.0 \%$ \\
\hline year 4 & $-4.0 \%$ & $-6.0 \%$ & $6.0 \%$ & $4.0 \%$ & $-5.0 \%$ & $-3.0 \%$ & $3.0 \%$ & $5.0 \%$ \\
\hline year 5 & $-4.0 \%$ & $-7.5 \%$ & $7.5 \%$ & $4.0 \%$ & $-5.0 \%$ & $-4.0 \%$ & $4.0 \%$ & $5.0 \%$ \\
\hline Annual discount rate & & & & & & & & \\
\hline year 1 & $9.5 \%$ & $10.5 \%$ & $10.5 \%$ & $11.5 \%$ & $10.5 \%$ & $11.5 \%$ & $11.5 \%$ & $12.5 \%$ \\
\hline year 2 & $9.5 \%$ & $10.3 \%$ & $10.8 \%$ & $11.5 \%$ & $10.5 \%$ & $11.3 \%$ & $11.8 \%$ & $12.5 \%$ \\
\hline year 3 & $9.5 \%$ & $10.0 \%$ & $11.0 \%$ & $11.5 \%$ & $10.5 \%$ & $11.0 \%$ & $12.0 \%$ & $12.5 \%$ \\
\hline year 4 & $9.5 \%$ & $9.8 \%$ & $11.3 \%$ & $11.5 \%$ & $10.5 \%$ & $10.8 \%$ & $12.3 \%$ & $12.5 \%$ \\
\hline year 5 & $11.5 \%$ & $11.5 \%$ & $10.5 \%$ & $10.5 \%$ & $12.5 \%$ & $12.5 \%$ \\
\hline
\end{tabular}

(V.P: Very Pessimistic, P : Pessimistic, O.: Optimistic, V.O : Very Optimistic)

Fig. 11. Initial Data for Fuzzy NPV. 


\begin{tabular}{|c|c|c|c|c|c|c|c|c|c|}
\hline & & \multicolumn{4}{|c|}{ Investment $X$} & \multicolumn{4}{|c|}{ Investment $Y$} \\
\hline Year & & $L_{0}$ & $L_{1}$ & $U_{1}$ & $U_{0}$ & $L_{0}$ & $L_{1}$ & $U_{1}$ & $U_{0}$ \\
\hline 0 & Initial Invest. & 3500000 & 3500000 & 3500000 & 3500000 & 4625000 & 4625000 & 4625000 & 4625000 \\
\hline & Charges & 500000 & 550000 & 550000 & 600000 & 650000 & 750000 & 750000 & 850000 \\
\hline & Fuzzy CFO & -4100000 & -4050000 & -4050000 & -4000000 & -5475000 & -5375000 & -5375000 & -5275000 \\
\hline 1 & Quantity & 21000 & 21400 & 21600 & 22000 & 21000 & 22200 & 22600 & 23800 \\
\hline & Sale Price & 96 & 99 & 101 & 104 & 85.0 & 90.0 & 90.0 & 94.5 \\
\hline & Charges & 1008000 & 1144044 & 1221696 & 1372800 & 624750 & 789210 & 823770 & 1012095 \\
\hline & Discount Rate & $9.5 \%$ & $10.5 \%$ & $10.5 \%$ & $11.5 \%$ & $10.5 \%$ & $11.5 \%$ & $11.5 \%$ & $12.5 \%$ \\
\hline & Fuzzy CF1 & 576861 & 811678 & 938965 & 1168950 & 687027 & 1053121 & 1116404 & 1470000 \\
\hline 2 & Quantity & 22050 & 22800 & 23450 & 24200 & 22050 & 24530 & 25650 & 28320 \\
\hline & Sale Price & 92.0 & 97.0 & 103.0 & 108.0 & 80.0 & 89.1 & 90.9 & 99.0 \\
\hline & Charges & 1014300 & 1172148 & 1376750 & 1568160 & 617400 & 852393 & 955950 & 1261656 \\
\hline & Discount Rate & $9.5 \%$ & $10.3 \%$ & $10.8 \%$ & $11.5 \%$ & $10.5 \%$ & $11.3 \%$ & $11.8 \%$ & $12.5 \%$ \\
\hline & Fuzzy CF2 & 370359 & 680646 & 1022785 & 1333834 & 396914 & 984679 & 1195156 & 1790528 \\
\hline 3 & Quantity & 23150 & 24170 & 25570 & 26620 & 23150 & 27000 & 29250 & 33700 \\
\hline & Sale Price & 88.0 & 93.0 & 107.0 & 112.0 & 76.5 & 87.3 & 92.7 & 103.5 \\
\hline & Charges & 1018600 & 1168861 & 1586874 & 1788864 & 619841 & 907484 & 1125262 & 1569578 \\
\hline & Discount Rate & $9.5 \%$ & $10.0 \%$ & $11.0 \%$ & $11.5 \%$ & $10.5 \%$ & $11.0 \%$ & $12.0 \%$ & $12.5 \%$ \\
\hline & Fuzzy CF3 & 179149 & 483271 & 1177407 & 1495005 & 141448 & 876798 & 1319063 & 2125733 \\
\hline 4 & Quantity & 24300 & 25500 & 28000 & 29300 & 24300 & 29600 & 33500 & 40100 \\
\hline & Sale Price & 84.0 & 87.0 & 113.0 & 116.0 & 72.0 & 84.6 & 95.4 & 108.0 \\
\hline & Charges & 1020600 & 1131435 & 1866760 & 2039280 & 612360 & 951581 & 1342278 & 1948860 \\
\hline & Discount Rate & $9.5 \%$ & $9.8 \%$ & $11.3 \%$ & $11.5 \%$ & $10.5 \%$ & $10.8 \%$ & $12.3 \%$ & $12.5 \%$ \\
\hline & Fuzzy CF4 & 1242 & 229626 & 1400962 & 1654215 & -124397 & 731841 & 1491797 & 2494087 \\
\hline 5 & Quantity & 25500 & 26800 & 30800 & 32200 & 25500 & 32300 & 38500 & 47700 \\
\hline & Sale Price & 80.0 & 80.0 & 120.0 & 120.0 & 70.0 & 80.0 & 100.0 & 110.0 \\
\hline & Charges & 1020000 & 1072000 & 2217600 & 2318400 & 624750 & 969000 & 1636250 & 2361150 \\
\hline & Discount Rate & $9.5 \%$ & $9.5 \%$ & $11.5 \%$ & $11.5 \%$ & $10.5 \%$ & $10.5 \%$ & $12.5 \%$ & $12.5 \%$ \\
\hline & Fuzzy CF5 & -161546 & -42707 & 1666837 & 1806587 & -319722 & 525934 & 1748767 & 2805705 \\
\hline & $B F A N P V$ & -3133934 & -1887487 & 2156956 & 3458590 & -4693731 & -1202627 & 1496186 & 5411054 \\
\hline
\end{tabular}

Fig. 12. Basic Fuzzy NPV. 


\begin{tabular}{|l|l|r|r|r|r|r|r|r|r|}
\hline & & \multicolumn{5}{|c|}{ Investment $X$} & \multicolumn{5}{|c|}{ Investment $Y$} \\
\hline Year & & \multicolumn{1}{|c|}{$L_{0}$} & \multicolumn{1}{c|}{$L_{1}$} & \multicolumn{1}{c|}{$U_{1}$} & \multicolumn{1}{c|}{$U_{0}$} & \multicolumn{1}{c|}{$L_{0}$} & \multicolumn{1}{c|}{$L_{1}$} & \multicolumn{1}{c|}{$U_{1}$} & $U_{0}$ \\
\hline & Fuzzy CFO & -4100000 & -4050000 & -4050000 & -4000000 & -5475000 & -5375000 & -5375000 & -5275000 \\
\hline & Fuzzy CF1 & 609164 & 841542 & 902878 & 1134366 & 789697 & 1069265 & 1074061 & 1351774 \\
\hline & Fuzzy CF2 & 431400 & 800295 & 891524 & 1264603 & 588523 & 1058264 & 1142559 & 1539007 \\
\hline & Fuzzy CF3 & 258109 & 751722 & 898461 & 1398930 & 391962 & 1060110 & 1179391 & 1746955 \\
\hline & Fuzzy CF4 & 94987 & 702297 & 905899 & 1531515 & 182549 & 1056750 & 1285240 & 1958849 \\
\hline & Fuzzy CF5 & -60321 & 631314 & 912653 & 1657339 & 3155 & 856627 & 1359828 & 2156117 \\
\hline & & & & & & & & & \\
\hline & IFA NPV & -2766661 & -322830 & 461414 & 2986753 & -3519115 & -273984 & 666079 & 3477702 \\
\hline
\end{tabular}

Fig. 13. IFA NPV.

The BFA approach generalizes that analysis. Namely, both projects can be compared according to different levels of possibility (called alpha-cuts). For instance, if we compare the intervals at the fully possible level (alpha cut $=1$ ), then we notice the following fact: project $X$ is more risky $\left(L_{1} X=-1887487\right.$ and $\left.L_{1} Y=-1202627\right)$, but may generate a higher gain $\left(U_{1} X=2156956\right.$ and $\left.U_{1} Y=1496186\right)$.

To globally compare two TFNs, various techniques may be used. The "defuzzification" methods are simple to apply. It deals with the reducing of information represented by the TFN into one average value, for instance the Center of Gravity, $(C o G=(L 0+L 1+$ $U 1+U 0) / 4)$. That approach gives 148532 euros for $X$ and 252720 euros for $Y$, which implies an advantage for project $Y$. However, project $Y$ is globally more risky than project $X$ (uncertainty measured by the Area Entropy of each $T F N: A E Y=6401799$ pour $A E X=5318$ 484). Therefore, the BFA approach confirms the classical analysis, with the advantage of continuous possible scenarios.

\section{Effect of the IFA approach on the choice of investment}

Figure 13 exposes the results issued by the IFA, taking into account relations more or less identified by managers. Differences with the BFA deal with the following points (as represented by Fig. 14):

- Decrease of imperfection: Area Entropy decreases by $38.5 \%$ for project $X$ and by

$38 \%$ for project $Y$.

- Suppression of the extreme scenarios: these values corresponded to combinations with no economic sense, as given the identified relations by the managers. This is specifically true for project $Y$, for which values $L_{0}$ and $U_{0}$ are strongly recentered.

- If project $Y$ remains more risky than project $X(A E X=3 \times 268 \times 829$ and $A E Y=3968440)$, the difference is not so high as with BFA outputs, and the whole $N P V$ is not any higher either $(C o G X=89669$ and $C o G Y=87670)$.

Hence, the IFA approach reduces the imprecision of the expected $N P V$, and strengthens the decision making by suppressing a great part of the artificial imperfection. Let us 


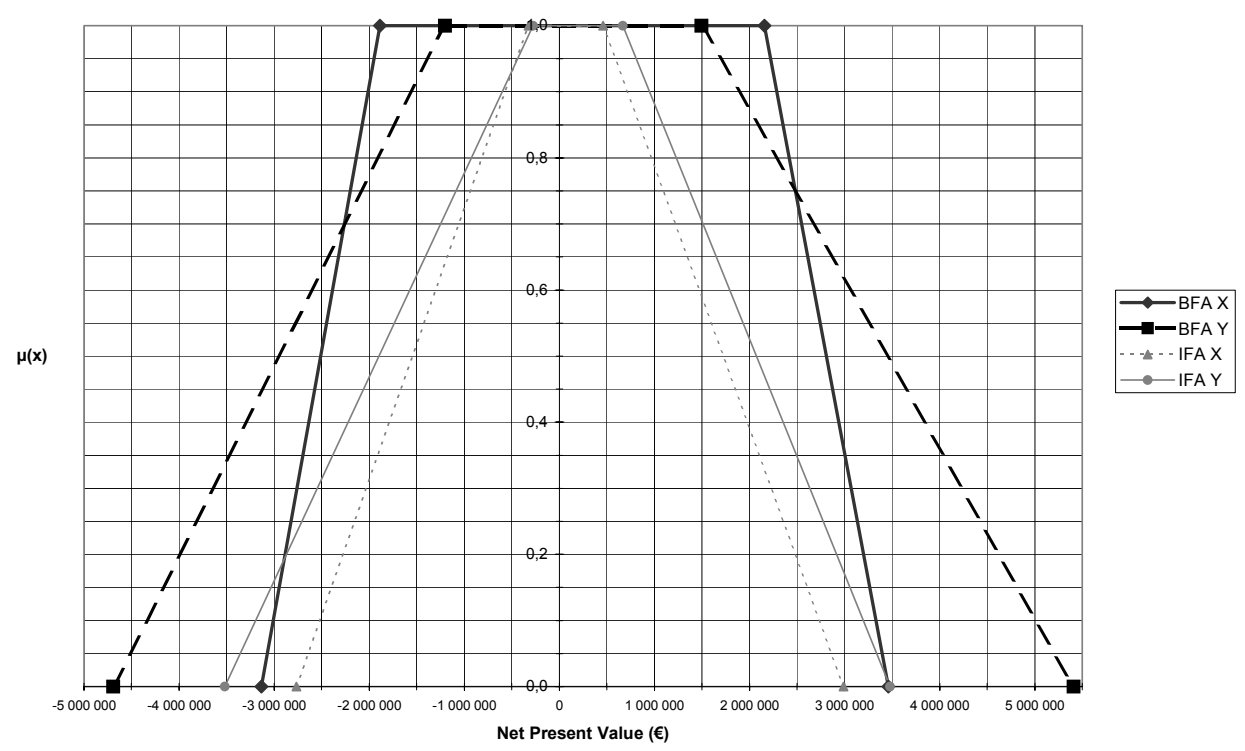

Fig. 14. Compared Net Present Value.

suppose that a maximal potential loss is adopted as a constraint on the choice of investment, which is a current practice in management. Then, if the limit stands at - 3.0 Meuros, then projects $X$ and $Y$ would have been rejected by the BFA approach, whereas the IFA makes clear that project $X$ does not overcome that constraint. Conversely, the high potential gains expected by project $Y$ were based on unrealistic combinations. Therefore, it seems reasonable that even a risk seeker may choose project $X$, because performance are quite similar, with less risky constraints (initial investment and expected sales lower, etc.).

Finally, the formula of the IFA enables the manager to reduce the gap between the higher and the lower intervals. Applied to the problematic of the investment decisionmaking based on the Net Present Value criterion, this ability tends to diminish the numbers of scenarios, thanks to the elimination of unrealistic scenarios. It generates savings in costs and time, as well as a facilitated decision-making.

\section{Conclusion}

In this paper, we present a new approach of fuzzy arithmetic. Based on the use of relations into arithmetic models, this Interactive Fuzzy Arithmetic enables us to decrease the imperfection of the output, when one of the basic operator $(+,-, \times, /)$ is used, which may be acute for decision making. We applied this approach to the Discounted Cash-Flows model to facilitate the choice of an investment, by diminishing the fuzziness of the Net Present Value. More generally, every arithmetic model in economic and management field may support the Interactive Fuzzy Arithmetic. 
However some limits remain, that will be further studied: for instance, the IFA approach may be adapted to take into account a specific relation: time. Namely, fuzzy dynamic models are now studied (e.g. Maeda et al., 1996; Virant, Zimic, 1996) because the time dimension is very important for management and economic purposes.

Finally, the approach proposed into this paper is a first proposal that should be further generalized to develop Interactive Fuzzy Arithmetic in order to implement models in real field of economics and management.

\section{References}

Aladenise N., Bouchon-Meunier B. (1997) Acquisition de connaissances imparfaites : mise en évidence d'une fonction d'appartenance, Revue internationale de systémique 11, (1), pp. 109127.

Buckley J.J. (1987) The fuzzy mathematics of finance, Fuzzy Sets and Systems 21, pp. 257-273.

Dubois D., Prade H. (1985) Fuzzy numbers: An overview, analysis of fuzzy information. In: Mathematics and Logic, Vol. 1. CRC Press, Boca Raton, Florida, pp. 3-39.

Gil Aluja J. (1995) Towards a new concept of economic research, Fuzzy Economic Review 0, pp. 5-24.

Frénois J.-P. ( 2001) L'analyse du risque dans les décisions financières, Gestion 25, pp. 13-26.

Karsak E.E., Tolga E. (2001) Fuzzy multi-criteria decision-making procedure for evaluating advanced manufacturing system investments, International Journal of Production Economics 69, pp. 49-64.

Klir G., Yuan B. (1995) Fuzzy Sets and Fuzzy Logic, Theory and Applications. Prentice Hall, New York.

Lesage C. (2000) Proposal for an interactive fuzzy arithmetic. In: Actes du Colloque Approches Connexionnistes en Sciences Économiques et de Gestion, Septième Rencontre Internationale, 14-15 December 2000, Paris, pp. 23-31.

Maeda H., Asaoka S., Murakami S. (1996) Dynamical fuzzy reasoning and its application to system modelling, Fuzzy Sets and Systems 80, pp.101-109.

Virant J., Zimic N. (1996) Attention to time in fuzzy logic, Fuzzy Sets and Systems 82, pp. 9-49.

Zadeh L.A. (1997) Toward a theory of fuzzy information granulation and its centrality in human reasoning and fuzzy logic, Fuzzy Sets and Systems 90, pp. 111-127.

Zadeh L.A. (1965) Fuzzy sets, Information and Control 8, pp. 338-353. 